\title{
Anlotinib combined with durvalumab in a patient with recurrent multifocal brain metastases of small cell lung cancer after definitive concurrent chemoradiotherapy and palliative radiotherapy of the lung and brain: a case report
}

\author{
Yuqi Wu ${ }^{1}$, Tao Zhang ${ }^{1}$, Yutao Liu ${ }^{2}$, Jianyang $\mathrm{Wang}^{1}, \mathrm{Nan}^{\mathrm{Bi}}{ }^{1}$ \\ ${ }^{1}$ Department of Radiation Oncology, National Cancer Center/National Clinical Research Center for Cancer/Cancer Hospital, Chinese Academy of \\ Medical Science and Peking Union Medical College, Beijing, China; ${ }^{2}$ Department of Oncology, National Cancer Center/National Clinical Research \\ Center for Cancer/Cancer Hospital, Chinese Academy of Medical Science and Peking Union Medical College, Beijing, China \\ Correspondence to: Prof. Nan Bi, MD, PhD. Department of Radiation Oncology, National Cancer Center/National Clinical Research Center for \\ Cancer/Cancer Hospital, Chinese Academy of Medical Science and Peking Union Medical College, No. 17 Panjiayuan Nanli, Chaoyang District, \\ Beijing 100021, China. Email: binan_email@163.com.
}

\begin{abstract}
The brain is a common metastatic site of small cell lung cancer (SCLC), but systematic treatment options are limited by the blood-brain barrier. Currently, the optimal treatment regimen remains controversial, especially for patients already treated by brain radiotherapy. Anlotinib is a novel oral multitarget tyrosine kinase inhibitor which has shown significant improvement in progression-free survival and overall survival in third-line or beyond therapy of advanced SCLC in a randomized, double-blind phase II study (ALTER1202 trial) based on a Chinese population sample. Emerging data has also suggested that immunotherapy, such as the programmed death ligand 1 (PD-L1) inhibitor, has a relatively high response rate in brain metastatic SCLC, although there is a lack of large sample-size studies. Integrating anlotinib and immunotherapy for recurrent or relapsing brain metastases (BMs) of SCLC has not been previously reported, but it is possible that these two treatments may have synergistic effects and provide even better outcomes. Here, we present a case of stage III SCLC who developed lung and BMs after concurrent chemoradiotherapy (cCRT) and achieved radiographic locally complete regression following whole brain irradiation (WBI) with a simultaneous integrated boost (SIB) technique. Durvalumab was delivered as maintenance therapy. Asymptomatic multifocal recurrence of BMs occurred after the administration of the second dose of durvalumab. After administration of combined durvalumab and anlotinib, the BMs achieved near-complete regression and no severe toxicity was reported. This suggests a potential synergistic effect of combined durvalumab and anlotinib in previously treated BMs in a patient with SCLC and may provide a direction for future clinical decisions.
\end{abstract}

Keywords: Small cell lung cancer (SCLC); case report; durvalumab; anlotinib; brain metastases (BMs)

Submitted Oct 16, 2020. Accepted for publication Feb 03, 2021.

doi: 10.21037/apm-20-2390

View this article at: http://dx.doi.org/10.21037/apm-20-2390

\footnotetext{
$\wedge$ ORCID: 0000-0001-6244-0931.
} 


\section{Introduction}

Small cell lung cancer (SCLC), a highly aggressive subtype, represents approximately $15 \%$ of all lung cancers (1). Although the disease is sensitive to platinumbased chemotherapy and radiotherapy, more than half of patients will experience rapid onset of brain metastases (BMs) during the course of the disease.

Few specific studies have been conducted with patients suffering from continued progression in the brain after receiving whole brain radiotherapy (WBRT). Regimens for SCLC subsequent systemic therapy are also limited for patients on maintenance immunotherapy who experience a relapse in the disease.

Here, we report a case with a background history of previous brain and lung metastases both treated by radiotherapy following concurrent chemoradiotherapy (cCRT), who had complete regression. During maintenance durvalumab treatment, the patient developed asymptomatic multi-foci BMs, which became a quite challenging situation after failure of multiple treatments. Disease progression became well-controlled after adding anlotinib dramatically. We present the following article in accordance with the CARE reporting checklist (available at http://dx.doi. org/10.21037/apm-20-2390).

\section{Case presentation}

A 57-year-old man was found to have a mediastinal lesion during his annual physical examination in December 2018. The patient had a 60 pack-year smoking history, as well as a 6-year history of hypertension. His uncle died from pancreatic cancer.

Endobronchial ultrasound-guided transbronchial needle aspiration (EBUS-TBNA) was performed a month later. The biopsy specimen of the subcarinal lymph node showed evidence of small cell carcinoma, and SCLC was suspected. Further immunohistochemical staining was recommended but was unsuccessful due to the limited sample size. Positron-emission tomography/computed tomography (PET-CT) revealed an enlarged subcarinal lymph node $(4.7 \mathrm{~cm} \times 3.0 \mathrm{~cm})$, with the maximum standard uptake value (SUVmax) of 18F-fluorodeoxyglucose (18F-FDG) being 7.4 (Figure 1). A solid nodule in the lower lobe of the right lung $(0.6 \mathrm{~cm} \times 0.5 \mathrm{~cm})$ and multiple small lymph nodes in the mediastinum $(2 \mathrm{R}, 4 \mathrm{R})$ and ipsilateral hilar with a slight FDG uptake were also detected. No evidence of metastasis was found by contrast magnetic resonance imaging (MRI) of the brain. SCLC was diagnosed, and the clinical stage was categorized as T1N2M0 IIIA according to the American Joint Committee on Cancer (AJCC), eighth edition.

The patient initially received inductive chemotherapy with etoposide and cisplatin for two cycles. Evaluation by CT scan showed a partial response (PR) based on the Response Evaluation Criteria in Solid Tumors (RECIST), version 1.1. Definitive cCRT was sequentially delivered, followed by one more cycle as adjuvant chemotherapy. The response was categorized as stable disease (SD) (Figure 2).

Three months after completing chemotherapy, the disease progressed quickly. A brain MRI found two new lesions, and BMs were suspected (Figure $3 A$ ). Whole brain irradiation (WBI) with a simultaneous integrated boost (SIB) (95\% PGTV 54 Gy/3 Gy/18 f, 95\% PTV 36 Gy/ 2 Gy/18 f) was delivered in November 2019, which had achieved radiographic complete regression (Figure 3B). Meanwhile, a CT scan discovered a newly identified nodule sized $1.1 \mathrm{~cm} \times 0.6 \mathrm{~cm}$ in the left upper lobe (Figure 4A). Subsequent tests showed the lesion in the left upper lobe had increased to $2.5 \mathrm{~cm} \times 2.4 \mathrm{~cm}$ one month after finishing brain irradiation (Figure $4 B$ ). Therefore, the patient subsequently received palliative radiation therapy up to a dose of 57 Gy administered in 19 fractions for lung metastasis (Figure 4C). One month later, in March 2020, the patient received his first dose of durvalumab $(10 \mathrm{mg} / \mathrm{kg}, \mathrm{q} 2 \mathrm{w})$ as maintenance therapy.

Unfortunately, the patient suffered from multifocal recurrence of BMs 3 days after the administration of the second dose of durvalumab (Figure $5 A$ ). He was referred for combination therapy of durvalumab (1,500 $\mathrm{mg} \mathrm{d} 1 / \mathrm{q} 4 \mathrm{w})$ and anlotinib (12 $\mathrm{mg} \mathrm{d} 1-\mathrm{d} 14 / \mathrm{q} 3 \mathrm{w})$ on the following day which has continued till now. Brain MRI showed little evidence of residual brain metastatic foci just 3 weeks after the beginning of the anlotinib treatment (Figure 5B), and a routine CT chest and abdomen scan showed no signs of recurrence. No severe toxicity was reported by the patient during follow-up.

All procedures performed in studies involving human participants were in accordance with the Helsinki Declaration (as revised in 2013) and the ethical standards of the institutional research committee. Written informed consent was obtained from the patient for publication of this study and any accompanying images.

\section{Discussion}

This patient was treated with inductive chemotherapy and 


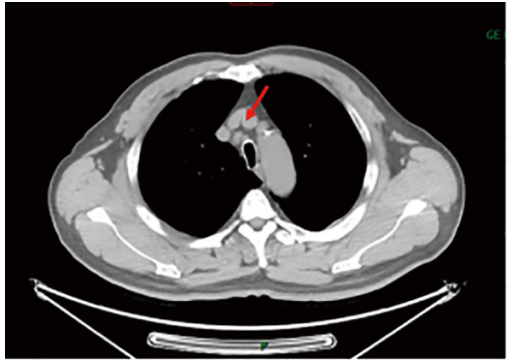

1-Upper paratracheal lymph node $(2 \mathrm{R})$

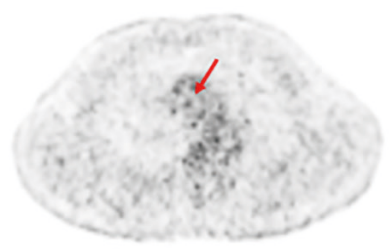

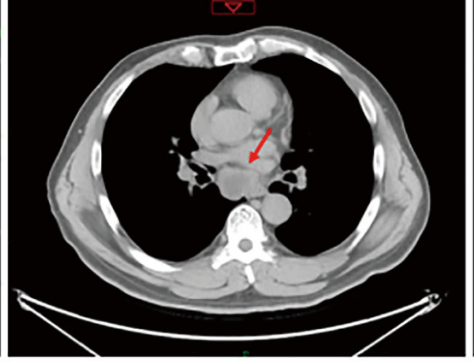

1-subcarinal lymph node

(7)

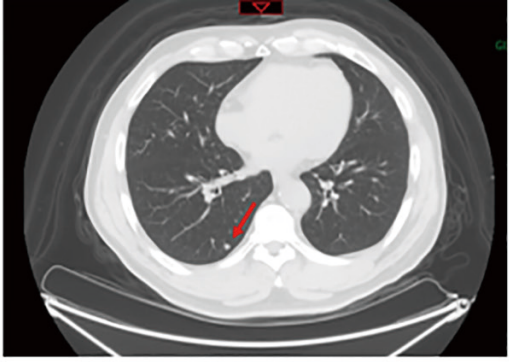

Primary site in right lower lobe
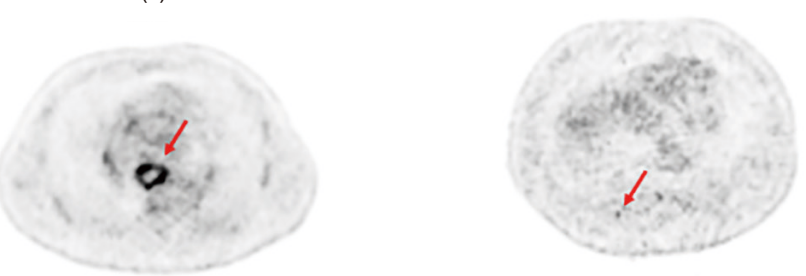

Figure 1 Positron emission tomography/computed tomography (PET-CT) image of primary tumor.

then concurrent chemotherapy as limited-stage SCLC, as recommended by guidelines. Immune maintenance therapy in limited-stage SCLC is not routinely recommended. Therefore, this patient did not receive immunotherapy after concurrent chemotherapy. To the best of our knowledge, there are many ongoing studies focusing on immune maintenance therapy in limited-stage SCLC, including ADRIATIC, STIMULI, ACHILES, etc. The best time and duration remain unclear. Prophylactic cranial irradiation (PCI) was suggested after cCRT but refused by the patient. Metastases in lung and brain were then occurred and controlled by radiotherapy. Based on available published trials of SCLC, durvalumab was chosen for the patient's treatment with atezolizumab as a feasible alternative. Since options were quite limited when new brain metastases occurred, adding anlotinib was tried. The integration regimen turned out to be effective and well-tolerated. Follow-up every 12 weeks for at least 2 years was scheduled.

Platinum-based doublets chemotherapy is considered to be the standard systemic treatment for SCLC, while radiation therapy is the primary choice for BMs due to the existence of the blood-brain barrier. Despite the encouraging results of programmed cell death protein 1 (PD-1)/programmed death ligand 1 (PD-L1) inhibitors in lung cancer treatment, BMs have not been a focus in many related clinical trials. In recent years, several cohort studies have specifically focused on the efficacy of PD-1/PD-L1 inhibitors for BMs in non-small cell lung cancer (NSCLC), and have revealed that the intracranial objective response rate (ORR) ranges from $17 \%$ to $33.3 \%$ (2-4). Immune checkpoint inhibitor (ICI)-treated NSCLC patients with BMs were found to have comparable survival rates to those without BMs $(2,5)$, sparking hope for the stillunderrepresented SCLC patients with BMs. Meanwhile, unlike the primary site, PD-L1 expression is commonly found in both BM tumor cells and tumor-infiltrating lymphocytes, which further encourages the use of PD-1/PD-L1 inhibitors $(6,7)$.

Durvalumab is one of the anti-programmed cell death ligant-1 agents. In the phase III CASPIAN study, durvalumab plus platinum etoposide in the first-line treatment of extensive-stage SCLC (ES-SCLC) significantly prolonged the median overall survival compared with platinum etoposide alone (13.0 vs. 10.3 months, HR $0.73, \mathrm{P}=0.0047)(8)$. This finding was consistent with the Impower133 study, in which atezolizumab improved both overall survival and progression-free survival as a first-line treatment of ES-SCLC when combined with carboplatin and etoposide (9). In this case, considering its highly invasive potential, the disease was treated with durvalumab as maintenance therapy following cCRT at the thoracic primary site and radiation therapy for the brain and lung metastases.

Anlotinib is a novel tyrosine kinase inhibitor which targets the vascular endothelial growth factor receptor (VEGFR), fibroblast growth factor receptor (FGFR) and other tyrosine kinase-mediated pathways. In recent years, the efficacy of anlotinib in advanced SCLC has 

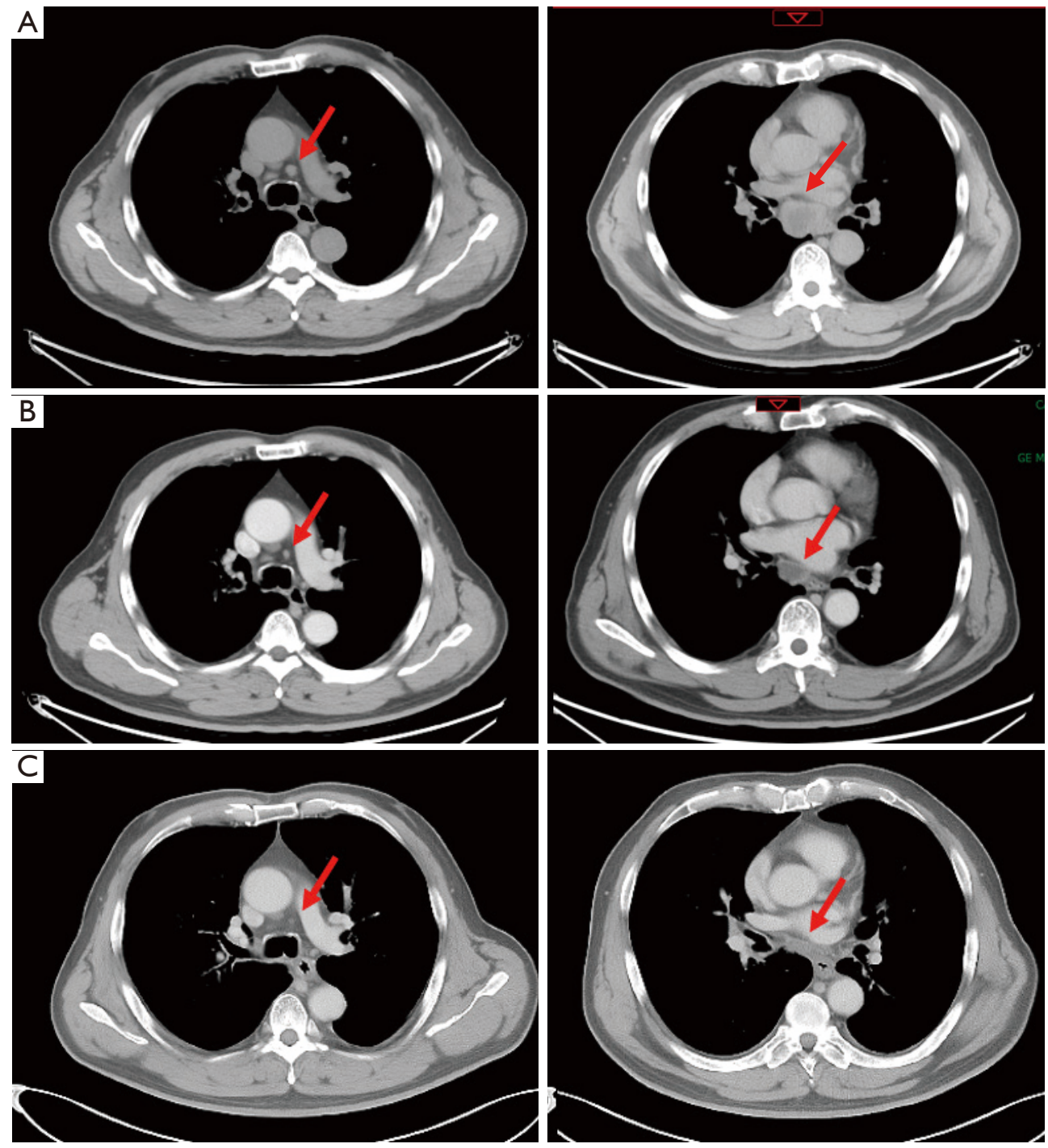

Figure 2 Chest computed tomography images of primary involved mediastinal lymph nodes at different time points. (A) Base line. (B) After induction chemotherapy for 2 cycles. (C) One month after concurrent chemoradiotherapy.

been demonstrated by several clinical trials. Results of the ALTER1202 trial, which included SCLC patients who had received at least 2 previous lines of chemotherapy, showed that the median disease-free survival (DFS) of the anlotinib group was 4.1 months, compared with 0.7 months for the placebo group (HR 0.19, $\mathrm{P}<0.0001$ ). In particular, patients with BMs had an absolute benefit of 3 months (3.8 vs. 0.8 months, HR $0.15, \mathrm{P}=0.003$ ) in the subgroup analysis. Anlotinib was also associated with a significant improvement in overall survival (7.3 vs. 4.9 months, HR 0.53 , $\mathrm{P}=0.0029)$ (10). A subgroup analysis of patients who previously received chemotherapy revealed similar results (11).
In addition to antiangiogenic effects, VEGF inhibitors may function as immunomodulators and augment the efficacy of immunotherapy (12). The phase 3 randomized trial, Impower 150, enrolled stage IV or metastatic nonsquamous NSCLC patients and assigned them in a 1:1:1 ratio to receive either combined $\mathrm{ABCP}(\mathrm{A}=$ atezolizumab, $\mathrm{B}=$ bevacizumab, $\mathrm{C}$ = carboplatin, $\mathrm{P}=$ paclitaxel), $\mathrm{ACP}$, or BCP (13). The primary endpoints were PFS and OS in the population without EGFR or ALK genomic alterations. The median PFS and OS was 8.3 and 19.2 months in the ABCP group, respectively, vs. 6.8 and 14.7 months in the BCP group, respectively. A phase 1 trial of sintilimab plus 

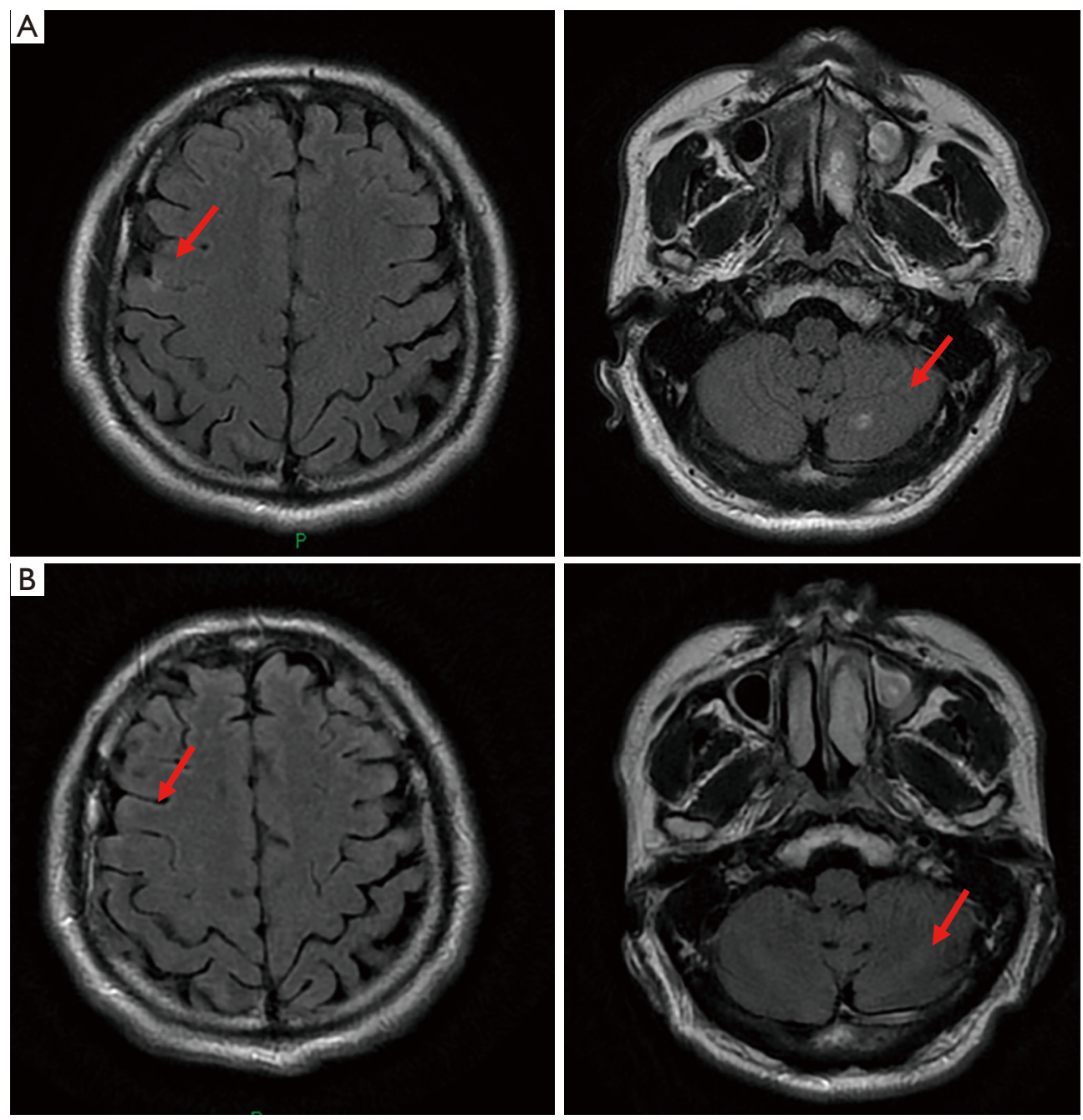

Figure 3 Brain magnetic resonance imaging images at different time points. (A) Five months after concurrent chemoradiotherapy, or 3 months after the end of adjuvant chemotherapy. Brain metastases are newly diagnosed. (B) One month after brain radiation therapy.
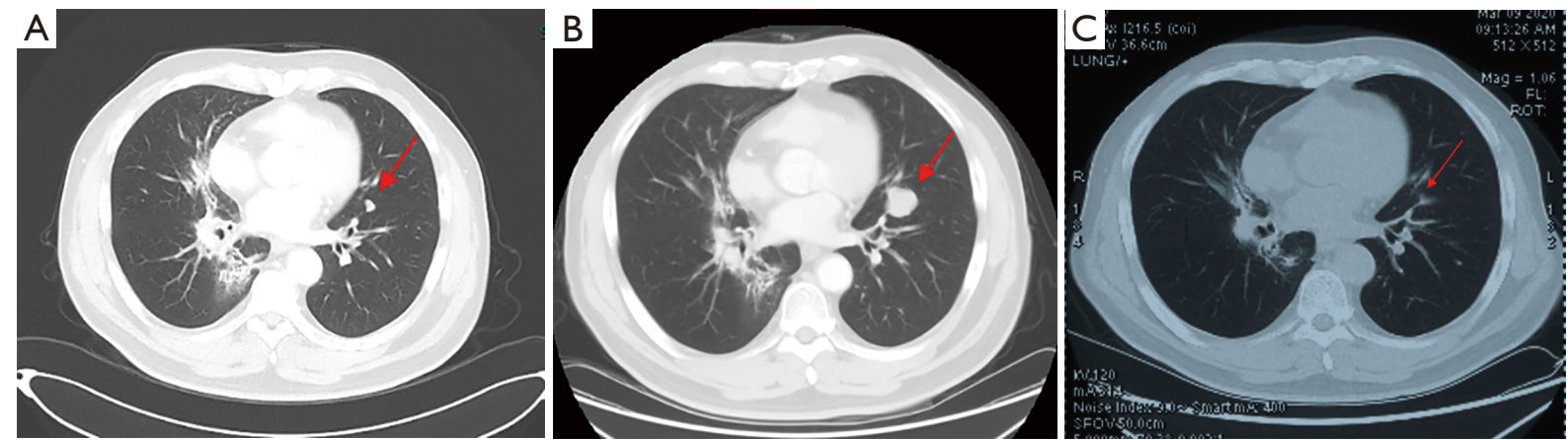

Figure 4 Chest computed tomography images of lung metastasis at different time points. (A) Five months after concurrent chemoradiotherapy, or 3 months after adjuvant chemotherapy ( 5 th cycle in total). A new lesion in the left upper lobe is found $(1.1 \mathrm{~cm} \times 0.6 \mathrm{~cm})$. (B) One month after brain radiation therapy. The lesion has increased to $2.5 \mathrm{~cm} \times 2.4 \mathrm{~cm}$ in diameter of the maximum section. (C) After radiotherapy for lung metastasis. 

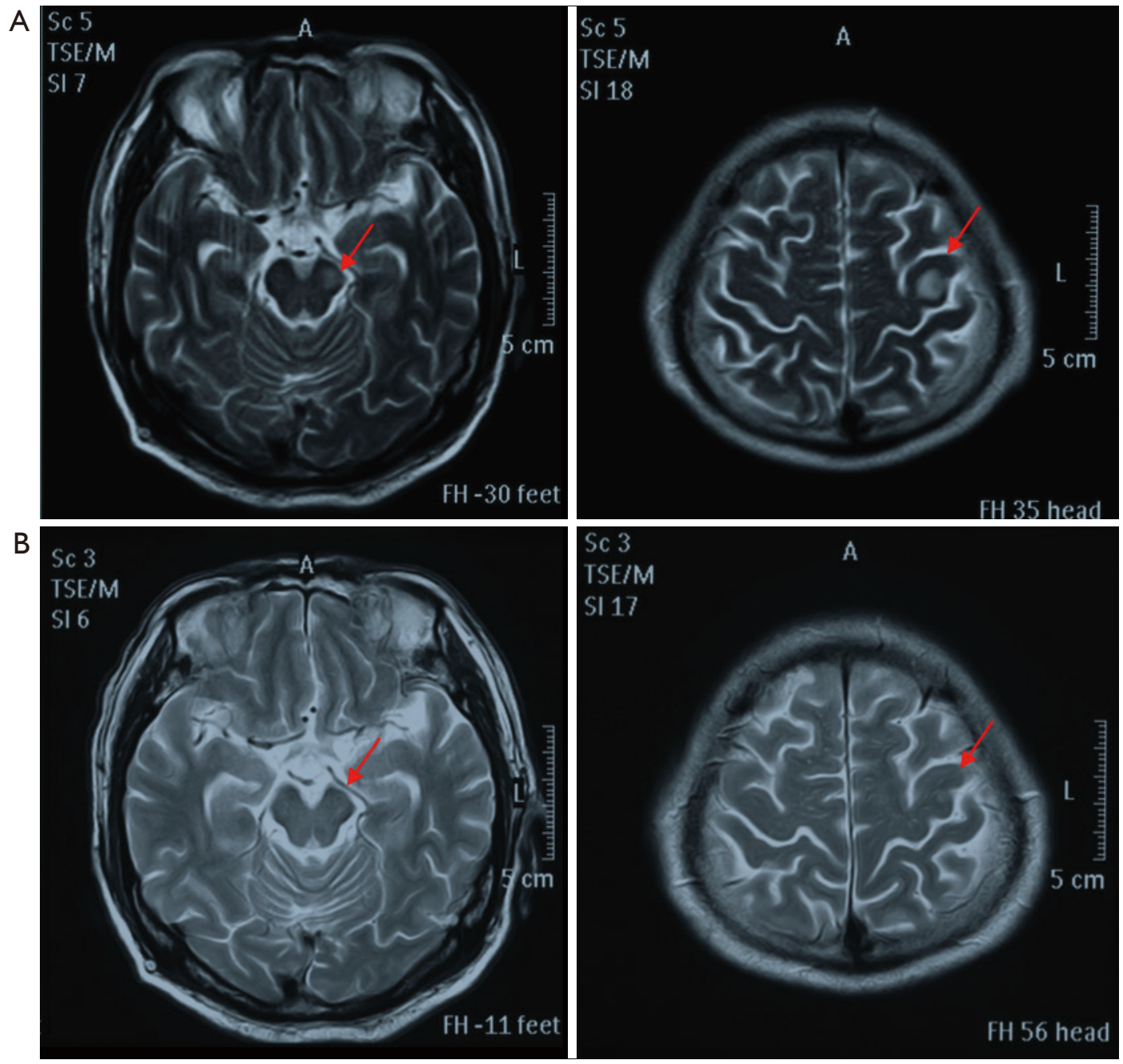

Figure 5 Brain magnetic resonance imaging images at different time points. (A) Recurrence in brain during durvalumab maintenance therapy, or 4 months after brain radiation therapy. (B) Three weeks after initiation of anlotinib combined with durvalumab.

anlotinib as a first-line treatment for advanced NSCLC showed consistent evidence with an ORR of $72.7 \%$ $(49.8-89.3 \%)$ and a disease control rate (DCR) of $100 \%$ (84.6-100\%) (2019 WCLC). In the present case study, the patient developed secondary BMs approximately 2 weeks after initiation of durvalumab treatment. With reference to similar reports from previous studies, we considered this could reflect a case of pseudoprogression $(14,15)$. Given the lack of supporting data in the treatment of BMs of SCLC, anlotinib was added into the ongoing immunotherapy as a trial, and the dose of durvalumab was adjusted correspondingly.

In the past few years, immune checkpoint inhibitors had shown promising effects in the treatment of SCLC.
Nevertheless, there are still many challenges, such as limited efficacy for intracranial metastases. Targeted therapy, such as anlotinib, had shed light on the treatment of pretreated SCLC. But more solid evidences are in need. More clinical trials on new drugs and new combination strategies are ongoing. For example, the ORR of durvalumab in combination with olaparib in patients with relapsed SCLC was $10.5 \%$ in a phase II study (16). Alisertib, an aurora A kinase inhibitor, plus paclitaxel potentially prolonged median PFS (3.32 vs. $2.17 \mathrm{~m}, \mathrm{HR}=0.77,95 \%$ CI: $0.557-1.067, \mathrm{P}=0.113$ ) compared with paclitaxel plus placebo as second-line therapy in a randomized phase II study (17). Adverse effects should also be carefully evaluated and compared in future clinical trials. 
A combined anlotinib and durvalumab approach showed a surprisingly promising result and was well-tolerated in this single case. Although no conclusion can be drawn, this may suggest a synergistic effect of an ICI and TKI in advanced SCLC with BMs. Further prospective clinical trials investigating the optimal strategy for SCLC patients are needed in the future.

\section{Acknowledgments}

Funding: Beijing Hope Run Special Fund of Cancer Foundation of China (LC2018A04).

\section{Footnote}

Reporting Checklist: The authors have completed the CARE reporting checklist. Available at http://dx.doi.org/10.21037/ apm-20-2390

Conflicts of Interest: All authors have completed the ICMJE uniform disclosure form (available at http://dx.doi. org/10.21037/apm-20-2390). The authors have no conflicts of interest to declare.

Ethical Statement: The authors are accountable for all aspects of the work in ensuring that questions related to the accuracy or integrity of any part of the work are appropriately investigated and resolved. All procedures performed in studies involving human participants were in accordance with the Helsinki Declaration (as revised in 2013) and the ethical standards of the institutional research committee. Written informed consent was obtained from the patient for publication of this study and any accompanying images.

Open Access Statement: This is an Open Access article distributed in accordance with the Creative Commons Attribution-NonCommercial-NoDerivs 4.0 International License (CC BY-NC-ND 4.0), which permits the noncommercial replication and distribution of the article with the strict proviso that no changes or edits are made and the original work is properly cited (including links to both the formal publication through the relevant DOI and the license). See: https://creativecommons.org/licenses/by-nc-nd/4.0/.

\section{References}

1. van Meerbeeck JP, Fennell DA, De Ruysscher DKM.
Small-cell lung cancer. Lancet 2011;378:1741-55.

2. Hendriks LEL, Henon C, Auclin E, et al. Outcome of Patients with Non-Small Cell Lung Cancer and Brain Metastases Treated with Checkpoint Inhibitors. J Thorac Oncol 2019;14:1244-54.

3. Crinò L, Bronte G, Bidoli P, et al. Nivolumab and brain metastases in patients with advanced non-squamous nonsmall cell lung cancer. Lung Cancer 2019;129:35-40.

4. Goldberg SB, Gettinger SN, Mahajan A, et al. Pembrolizumab for patients with melanoma or non-smallcell lung cancer and untreated brain metastases: early analysis of a non-randomised, open-label, phase 2 trial. Lancet Oncol 2016;17:976-83.

5. Zhang G, Cheng R, Wang H, et al. Comparable outcomes of nivolumab in patients with advanced NSCLC presenting with or without brain metastases: a retrospective cohort study. Cancer Immunol Immunother 2020;69:399-405.

6. Berghoff AS, Ricken G, Wilhelm D, et al. Tumor infiltrating lymphocytes and PD-L1 expression in brain metastases of small cell lung cancer (SCLC). J Neurooncol 2016;130:19-29.

7. Iams WT, Porter J, Horn L. Immunotherapeutic approaches for small-cell lung cancer. Nat Rev Clin Oncol 2020;17:300-12.

8. Paz-Ares L, Dvorkin M, Chen Y, et al. Durvalumab plus platinum-etoposide versus platinum-etoposide in firstline treatment of extensive-stage small-cell lung cancer (CASPIAN): a randomised, controlled, open-label, phase 3 trial. Lancet 2019;394:1929-39.

9. Horn L, Mansfield AS, Szczęsna A, et al. First-Line Atezolizumab plus Chemotherapy in Extensive-Stage Small-Cell Lung Cancer. N Engl J Med 2018;379:2220-9.

10. Yang $S$, Zhang Z, Wang Q. Emerging therapies for small cell lung cancer. J Hematol Oncol 2019;12:47.

11. Wang Q, Cheng Y, Li K, et al. OA03.02 Effect of Anlotinib in Advanced Small Cell Lung Cancer Patients Previously Received Chemoradiotherapy: A Subgroup Analysis in ALTER 1202 Trial. J Thorac Oncol 2019;14:S211.

12. Hegde PS, Wallin JJ, Mancao C. Predictive markers of anti-VEGF and emerging role of angiogenesis inhibitors as immunotherapeutics. Semin Cancer Biol 2018;52:117-24.

13. Socinski MA, Jotte RM, Cappuzzo F, et al. Atezolizumab for First-Line Treatment of Metastatic Nonsquamous NSCLC. N Engl J Med 2018;378:2288-301.

14. Galldiks N, Kocher M, Ceccon G, et al. Imaging challenges of immunotherapy and targeted therapy in 
patients with brain metastases: response, progression, and pseudoprogression. Neuro Oncol 2020;22:17-30.

15. Melian M, Lorente D, Aparici F, et al. Lung brain metastasis pseudoprogression after nivolumab and ipilimumab combination treatment. Thorac Cancer 2018;9:1770-3.

16. Thomas A, Vilimas R, et al. Durvalumab in Combination with Olaparib in Patients with Relapsed
SCLC: Results from a Phase II Study. J Thorac Oncol 2019;14:1447-57.

17. Owonikoko TK, Niu H, et al. Randomized Phase II Study of Paclitaxel plus Alisertib versus Paclitaxel plus Placebo as Second-Line Therapy for SCLC: Primary and Correlative Biomarker Analyses. J Thorac Oncol 2020;15:274-87.

(English Language Editor: D. Fitzgerald)

Cite this article as: Wu Y, Zhang T, Liu Y, Wang J, $\mathrm{Bi}$ N. Anlotinib combined with durvalumab in a patient with recurrent multifocal brain metastases of small cell lung cancer after definitive concurrent chemoradiotherapy and palliative radiotherapy of the lung and brain: a case report. Ann Palliat Med 2021;10(2):2379-2386. doi: 10.21037/apm-20-2390 